\title{
Advanced neutral gas diagnostics for magnetic confinement devices
}

\author{
U. Wenzel ${ }^{\mathrm{a}}$, T. Kremeyer ${ }^{\mathrm{b}}$, G. Schlisio ${ }^{\mathrm{a}}$, M. Marquardt ${ }^{\mathrm{a}}$, T.S. Pedersen ${ }^{\mathrm{a}}$, O. Schmitz ${ }^{\mathrm{b}}$, \\ B. Mackie ${ }^{\mathrm{c}}$, J. Maisano-Brown ${ }^{\mathrm{d}}$, and the W7-X team ${ }^{\mathrm{a}}$ \\ ${ }^{a}$ Max-Planck-Institut für Plasmaphysik (IPP), EURATOM Association, Wendelsteinstr. \\ 1, 17491 Greifswald, Germany \\ ${ }^{\mathrm{b}}$ Department of Engineering Physics, University of Wisconsin, Madison, Wisconsin \\ 53706, USA \\ ${ }^{\mathrm{c}}$ APTech, 1600 NE Miller St., McMinnville, OR USA \\ ${ }^{\mathrm{d}}$ MIT Department of Physics, 77 Massachusetts Avenue, Cambridge, MA 02139-4307
}

July 5, 2017

\begin{abstract}
For the study of particle exhaust in nuclear fusion devices the neutral pressure must be measured in strong magnetic fields. We describe as an example the neutral pressure gauges in the Wendelstein 7-X stellarator. Two types are used: Hot cathode ionization gauges (or ASDEX pressure gauges) and Penning gauges. We show some results from the first experimental campaign. Main problems were runtime effects and the failure of some ASDEX pressure gauges. To improve the reliability we integrated a new $\mathrm{LaB}_{6}$ electron emitter into the ASDEX pressure gauges. A special Penning gauge without permanent magnets was developed in order to operate Penning gauges in the vacuum vessel, too. These new pressure gauges will be used in the upcoming campaign of Wendelstein 7-X.
\end{abstract}

\section{Introduction}

Wendelstein 7-X (W7-X) is a helical axis stellarator optimized for low neoclassical transport, a small bootstrap current and a good fast ion confinement [1]. The magnetic field is generated by 10 sets of 7 magnetic superconducting coils. W7-X is typically operated with a magnetic field of $2.5 \mathrm{~T}$ on the plasma axis corresponding to the central absorption of the second harmonic electronic cyclotron resonance heating (ECRH) at $140 \mathrm{GHz}$. In the initial experimental campaign in 2015/2016 the machine was equipped with five poloidal limiters. W7-X was operated in a special magnetic configuration with good flux surfaces in the scrape-off layer. Plasma breakdown and heating were provided by ECRH with an power up to $4.3 \mathrm{MW}$. Electron temperatures of about $7 \mathrm{keV}$, ion temperatures of about $2 \mathrm{keV}$ and electron densities of about $4 \times 10^{19} \mathrm{~m}^{-3}$ were achieved simultaneously. Plasmas of up to $6 \mathrm{~s}$ were sustained [2].

For the study of particle exhaust the neutral pressure must be measured in the plasma edge. Pressure measurements in strong magnetic fields were reviewed in [3]. In W7-X several types of pressure gauges are used. In the pumping ducts Penning gauges were installed with a time resolution of $1 \mathrm{~s}$. They are running continuously when the pumps are active. Inside the plasma vessel we use fast hot cathode ionization gauges for the measurement of the neutral pressure. They were developed on the ASDEX tokamak [4] and are called ASDEX pressure gauges (APG). 
They have a time resolution of $200 \mu \mathrm{s}$. A third system is a fast diagnostic Penning gauge which is completed by an optical system to measure partial pressures [5].

\section{Fast hot cathode ionization gauges}

APGs are the main pressure diagnostic inside the plasma vessel. The schematic of the APG and the applied potentials are shown in Fig. 1. The APG is seen from above (compare also the photograph of the APG in Fig. 3, left image). It is a tetrode in linear arrangement which has to be oriented parallel to the direction of the magnetic field $\mathrm{B}$ because the electrons from the filament can move only in the direction of this field (and not perpendicular). The potentials are: filament $+70 \mathrm{~V}$, acceleration grid $+250 \mathrm{~V}$, ion collector $0 \mathrm{~V}$. The control electrode is switched between +105 and $+20 \mathrm{~V}$ to chop the electron current in order to cancel large offsets and leak currents.

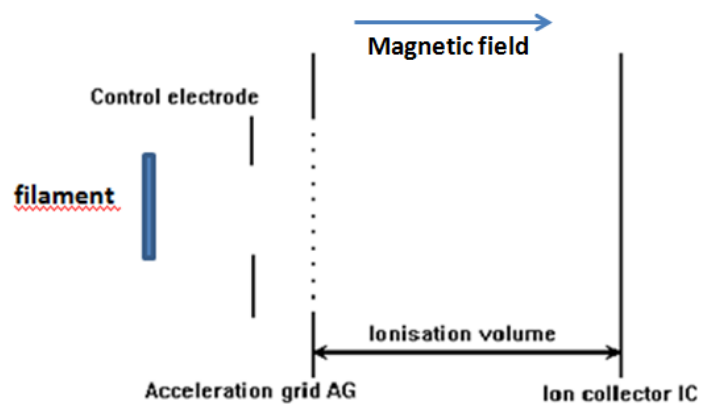

Figure 1: Schematic of the APG (left) and the potential distribution (right). The abcissa in the right image is the distance from the acceleration grid (in $\mathrm{mm}$ ). The four elements of the APG are the filament (cathode), control electrode (Wehnelt), acceleration grid (anode) and ion collector. The filament is on $70 \mathrm{~V}$ in order to allow the chopping of the electron emitter by the control electrode.

The electron current is measured at the acceleration grid and kept constantly by a feedback loop using a controllable power supply. The neutral pressure is proportional to the ion current which is measured at the ion collector. In contrast to standard ionization gauges, the APG uses a thick filament to withstand the strong $\mathrm{j}$ x B forces. We use thoriated tungsten wires (1.8\% ThO $\mathrm{O}_{2}$ ) with $0.6 \mathrm{~mm}$ diameter.

A set of five manometers of ASDEX type has been installed for the initial campaign of W7-X. We selected midplane positions $15 \mathrm{~cm}$ away from the plasma vessel contour in every of the five stellarator modules. For the control electrode, acceleration grid and ion collector we use minerally insulated cables inside the plasma vessel. For the filament copper cables are used. The APGs were mounted on the top of immersion systems for easy handling. These carrier structures are about $2 \mathrm{~m}$ long with the gauge on top and the vacuum flange on the other side. The gauge is mounted on an adjustment unit which allows to set it exactly parallel to the magnetic field. The APGs were calibrated in-situ by filling the plasma vessel with hydrogen or helium.

Fig.2 shows the gauges traces (electron and ion current) of a W7-X experimental programme using hydrogen as working gas. At $\mathrm{t}=0$ the programme is started by the ignition of the ECRH. Before the ignition the electron current feed-back loop ist started at $-10 \mathrm{~s}$. The electron current is ramped-up to the selected value of $200 \mu \mathrm{A}$. The ion current reacts first on the filling of the 


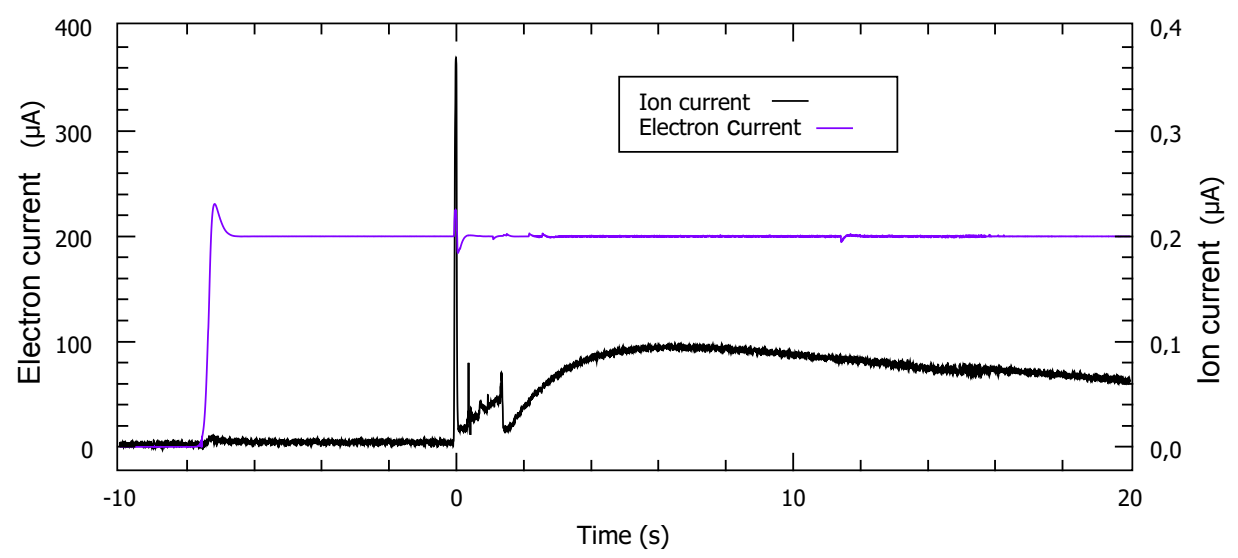

Figure 2: Electron (left axis) and ion current (right axis) of a mid-plane pressure gauge in module 4. From the ion current the pressure can be calculated. The experimental programme number is 2017-03-10.10. The electron current was held at $200 \mu \mathrm{A}$ by feedback control. The experiment was started at 0 and was finished at $1.4 \mathrm{~s}$. Note the large pressure rise after the experiment by outgassing of hydrogen from the inner walls.

plasma vessel with hydrogen shortly before $0 \mathrm{~s}$. The experimental programme ends at $1.4 \mathrm{~s}$. During the experiment the neutral density increases due to additional gas puffing in order to raise the electron density in the plasma. After the experiment the neutral density increases further due to the outgassing of neutral particles from the walls. For hydrogen the ion current can be converted to a neutral pressure using a quadratic polynomial with the coefficients $\mathrm{a}_{0}=$ $6.63 \mathrm{e}-9, \mathrm{a}_{1}=9.66 \mathrm{e}-5$ and $\mathrm{a}_{2}=4.251 \mathrm{e}-5$ ( $\mathrm{I}$ in $\mu A$, $\mathrm{p}$ in mbar).
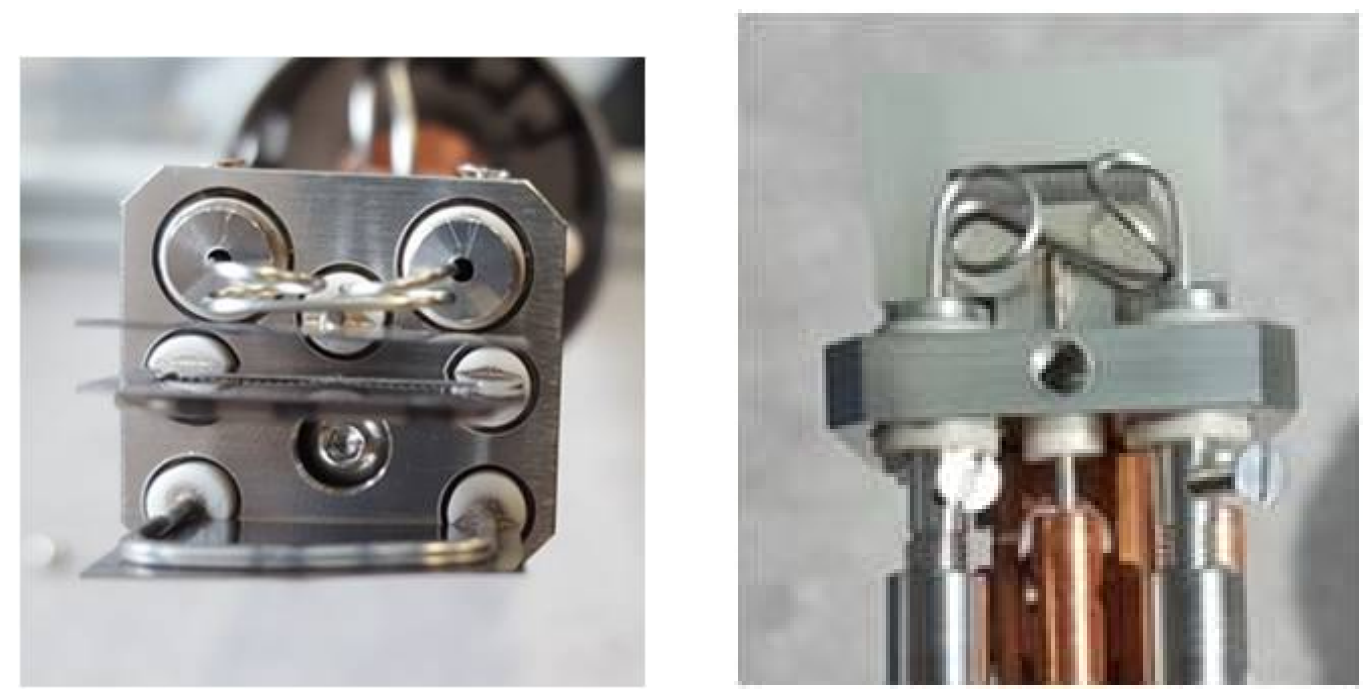

Figure 3: Deformation of the gauge filaments during the initial campaign of W7-X. The APG in module 4 was still functional (left image) while the APG in module 5 (right image) failed completely. 


\section{Reliability of the ASDEX pressure gauges}

During the initial experimental campaign we operated 4 APGs with a typical filament current of $18 \mathrm{~A}$. Two of them showed degradation effects toward the end of the campaign. The gauge in module 5 finally failed to reach the default value of the electron current and was shut-off. The gauge in module 4 was functional till the end of the campaign but had a higher power consumption. Visual inspection after the opening of the vacuum vessel showed a deformation of the filaments in both APGs that was caused by the strong $\mathrm{j} x \mathrm{~B}$ force. Fig. 3 showed that the filaments were deformed to different extents. The filament of the APG of module 5 (see the right image) was deformed so strongly that it was not longer functional. The loop appeared to be the cause of failure.

A degradation of the gauges was already observed earlier. In the predecessor device W7-AS 9 APGs were installed for the final divertor experiment. During the divertor campaign 2 of the 9 gauges failed.

In W7-X the degradation and the subsequent failure occured at the end of the campaign after 10 weeks of operation with 3 experimental days and 30 experiments per day. In each experiment we set an operational time of $20 \mathrm{~s}$. This gives a total operation time of about $5 \mathrm{~h}$ with the $\mathrm{jx}$ B force on the filament. During the next operational period of W7-X we will operate 18 APGs over a comparable time and expect some failures, too.

\section{Fast hot cathode ion gauges with $\mathrm{LaB}_{6}$ electron emittter}

In order to improve the reliability of the APGs, the tungsten filament was replaced by a $\mathrm{LaB}_{6}$ crystal rod. $\mathrm{LaB}_{6}$ has a very low electron work function, around $2.7 \mathrm{eV}$, and is widely used as a thermionic emitter. We expect a much lower heating current than $18 \mathrm{~A}$ for the same electron electron current of $200 \mu A$ and, thus, in the magnetic field much lower $\mathrm{j}$ x B forces on the crystal. Fig. 4 shows the $\mathrm{LaB}_{6}$ electron emitter that was integrated into the APG. Two molybdenum posts are soldered in a ceramic base. The base is formed so that it fits to the APG after same minor modifications. Between the posts the crystal and two blocks of pyrolitic graphite were mounted. Currents from 2 to $4 \mathrm{~A}$ heat the carbon blocks Ohmically and the blocks heat the crystal by heat conduction. Temperatures from 1600 to $1900 \mathrm{~K}$ were obtained in the middle of the crystal.

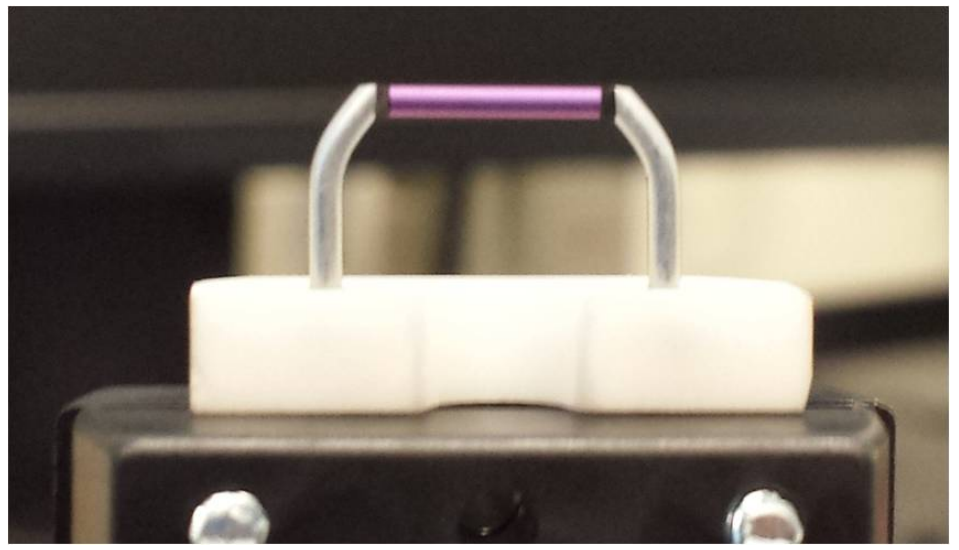

Figure 4: $\mathrm{LaB}_{6}$ electron emitter for the APGs. The pink $\mathrm{LaB}_{6}$ crystal has a length of $7 \mathrm{~mm}$ and a diameter of $1 \mathrm{~mm}$. It is clamped by two molybdenum posts (gray). The special ceramic base (the white part) fits into the APG after some modifications. 
Fig. 5 shows the first test results of the new pressure gauge without magnetic field. We applied the following potentials for the test: electron emitter $0 \mathrm{~V}$, control electrode $+35 \mathrm{~V}$ and acceleration grid $+180 \mathrm{~V}$, i.e. the relative potential distribution is the same as in the APGs. Fig. 5 shows the electron current measured at the acceleration grid as a function of the heating current.

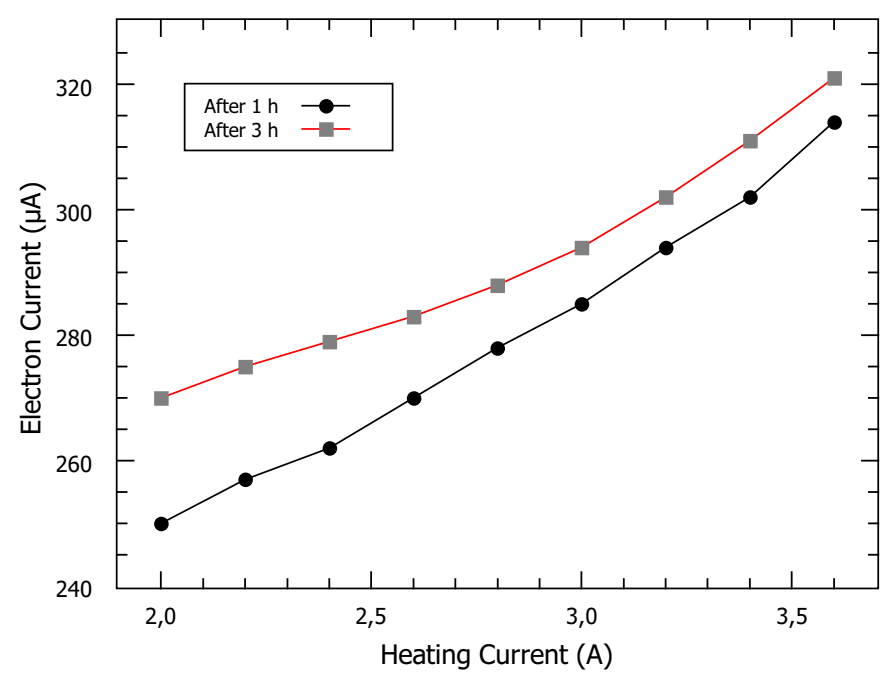

Figure 5: Electron current at the accelaration grid vs. heating current of the $\mathrm{LaB}_{6}$ crystal manometer. Currents from 250 to $320 \mu \mathrm{A}$ can be extracted from the $\mathrm{LaB}_{6}$ emitter.

The electron current increases almost linearly with the heating current. Currents from 250 to $320 \mu A$ can be extracted from the emitter. The increase is limited by the space-charge effect. The electron current increases a bit over time. The crystal seems to be not fully activated yet. For the pressure measurement the electron current must be feedback controlled. As expected we obtain with the $\mathrm{LaB}_{6}$ crystal electron currents in the same order of magnitude as with the APG but at much lower heating currents (almost one order of magnitude). Therefore, much lower $\mathrm{j} \times \mathrm{B}$ forces will act on the crystal in a magnetic field. The APG with $\mathrm{LaB}_{6}$ crystal will be tested in a superconducting magnet at $3.1 \mathrm{~T}$.

\section{$5 \quad$ Penning gauges}

In every pumping port Penning gauges are installed for a routine measurement of the vacuum pressure in the plasma vessel. Their time resolution is one second. They are positioned about 4.5 $\mathrm{m}$ away from plasma vessel midplane position because of there own magnetic fields. The stray field from $\mathrm{W} 7-\mathrm{X}$ is about $6 \mathrm{mT}$ at this position. Furthermore, a special diagnostic Penning gauge (a commercial Alcatel CF2P gauge) was installed at the end of the port AEE41. At the beginning of this port there is also an APG in the midplane position of module 4. Time resolution of both systems is comparable. The distance between them is only $2.2 \mathrm{~m}$, i.e. the stray field from W7-X is relatively large at the position of the diagnostic Penning gauge (about $100 \mathrm{mT}$ ) which can give rise to oscillations on the Penning gauge signal.

In Fig. 6 some selected neutral pressure gauges are compared. They show the reaction on a short gas filling pulse of $70 \mathrm{~ms}$ during a so called gas test. We find large runtime effects between this pressure gauges. The runtime effect between the APGs is in the order of hundreds of ms. The runtime effect between in-vessel APG and ex-vessel Penning gauges is in the order of seconds depending on the position. This delay is much larger than expected when only the thermal 


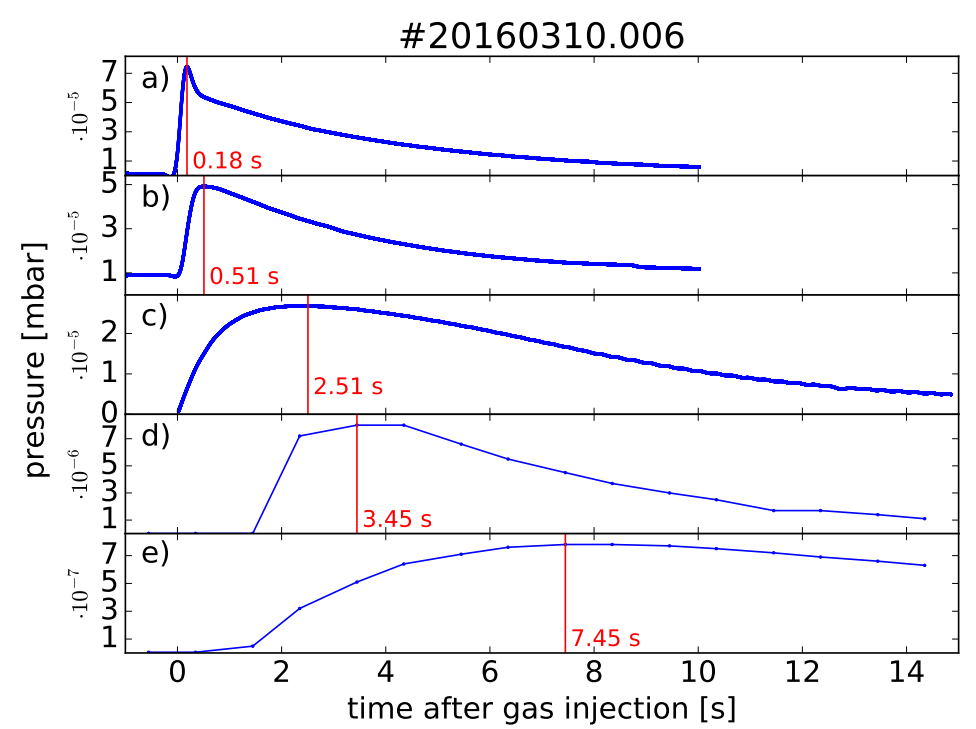

Figure 6: Comparison of the pressure measurements of a gas filling pulse of 70ms length: (a) APG module 4, (b) APG module 2, (c) diagnostic Penning gauge module 4, (d) Penning gauge pumping port module 4 and (e) Penning gauge pumping port module 2 . The red lines indicate the times of maximum pressure. The gas valve between module 5 and 4 was used.

velocity is taken into account. There seems to be also an intrinsic delay of the Penning gauges. To get rid of the large runtime effects as well as the stray field problems, a new Penning probe head has been developed. This probe head does not rely on permanent magnets but instead uses the magnetic field of W7-X. This allows the diagnostic to be mounted close to the plasma or inside of the divertor baffle. Several anode geometries were tested to allow for an optimized optical observation of the discharge in the Penning cell, while remaining a reliable total pressure measurement [6]. Fig. 7 shows the design of the Penning probe head that was selected for W7$\mathrm{X}$. The open geometry combined with a collimator lens behind a pin hole camera allows for an optimized intrinsic optical observation by a high temperature, vacuum fiber. The probe head is then mounted on the same adjustment unit as the APGs to adjust the probe head to the magnetic field as shown in Fig. 7.

We tested the new Penning probe head in a $3.1 \mathrm{~T}$ superconducting magnet. The current of the discharge in the Penning cell depends on the neutral pressure. Fig. 7 shows the measured pressure curve in helium with $2 \mathrm{kV}$ anode voltage. The discharge current increases exponentially in the range from $2 \times 10^{-4}$ mbar to $4 \times 10^{-3}$ mbar. By selection of other anode potentials the pressure range can be varied.

\section{Summary}

We have described the different neutral pressures gauges in W7-X and shed some light on the problems which arise from the strong magnetic field. For the APG the reliability is main problem. We developed a $\mathrm{LaB}_{6}$ crystal pressure gauge which can be operated at much lower heating currents. For the Penning gauges the own magnetic field prevents the application in the plasma vessel with the consequence of large runtime effects and loss of information. We developed a Penning cell without permanent magnets which can be operated in the vacuum vessel at $2.2 \mathrm{~T}$. Both pressure gauges will be tested in the strong magnetic field of W7-X during one of the next campaigns. 

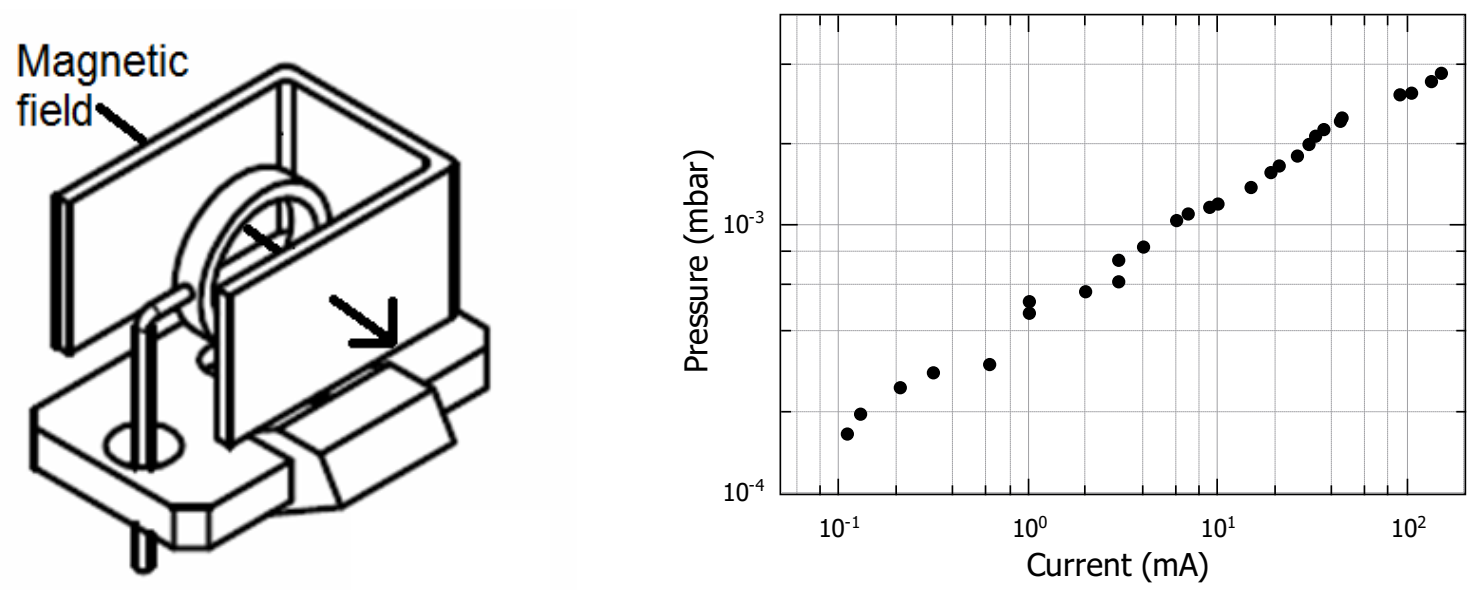

Figure 7: Penning cell geometry (left image) and pressure curve of the in-vessel Penning gauge in a $3.1 \mathrm{~T}$ magnetic field (right image). The pressure curve applies to $2 \mathrm{kV}$ anode voltage and helium.

\section{Acknowledgement}

This work has been carried out within the framework of the EUROfusion Consortium and has received funding from the Euratom research and training programme 2014-2018 under grant agreement No 633053. The views and opinions expressed herein do not necessarily reflect those of the European Commission.

\section{References}

[1] G. Grieger, W. Lotz, P. Merkel, J. Nührenberg, J. Sapper, E. Strumberger, H. Wobig, R. Burhenn, V. Erckmann, U. Gasparino, L. Giannone, H. J. Hartfuss, R. Jaenicke, G. Kühner, H. Ringler, A. Weller, F. Wagner, the W7-X Team, and the W7-AS Team. Physics optimization of stellarators. Physics of Fluids B: Plasma Physics, 4(7):2081-2091, 1992.

[2] T.S. Pedersen, A. Dinklage, Y. Turkin, R. Wolf, S. Bozhenkov, J. Geiger, G. Fuchert, H.S. Bosch, K. Rahbarnia, H. Thomsen, U. Neuner, T. Klinger, A. Langenberg, P. Trimino Mora, H.and Kornejew, J. Knauer, M. Hirsch, and the W7-X team. Key results from the first plasma operation phase and outlook for future performance in Wendelstein 7-X. submitted to Physics of Plasmas, 2017.

[3] H.F. Dylla. Pressure measurements in magnetic fusion devices. J. Vac. Sci. Technol., 20(2):119-128, 1982.

[4] G. Haas and H.-S. Bosch. In vessel pressure measurement in nuclear fusion experiments with asdex gauges. Vacuum, 51:39-46, 1998.

[5] T. Denner, K.H. Finken, and G. Mank. Helium partial pressure measurement in a deuterium environment. Review of scientific instruments, 67(10):3515-3520, 1996. 
[6] K. Flesch, T. Kremeyer, O. Schmitz, V. Soukhanovskii, and U. Wenzel. Development of miniaturized, spectroscopically assisted penning gauges for fractional helium and hydrogen neutral pressure measurements. Rev. Sci. Instrum., 87:11E529, 2016. 